\title{
A game theory approach for efficient power control and interference management in two-tier femtocell networks based on local gain
}

\author{
Y. A. Al-Gumaei ${ }^{1}$, K. A. Noordin ${ }^{1}$, A. W. Reza ${ }^{1}$ and K. Dimyati ${ }^{2}$ \\ ${ }^{1}$ Department of Electrical Engineering, Faulty of Engineering, University of Malaya \\ Kuala Lumpur, 50603 - Malaysia \\ [e-mail: wasif@um.edu.my] \\ ${ }^{2}$ Département of Electrical and Electronic Engineering, Faculty of Engineering, National Defence University of \\ Malaysia \\ Kuala Lumpur - Malaysia \\ [e-mail: kaharudin@upnm.edu.my] \\ *Corresponding author: A. W. Reza
}

Received January 26, 2015; revised May 18, 2015; accepted June 9, 2015;

published July 31, 2015

\begin{abstract}
In the recent years, femtocell technology has received a considerable attention due to the ability to provide an efficient indoor wireless coverage as well as enhanced capacity. However, under the spectrum sharing between femtocell user equipment (FUEs) and the owner of spectrum macrocell user equipment (MUEs), both may experience higher uplink interference to each other. This paper proposes a novel distributed power control algorithm for the interference management in two-tier femtocell networks. Due to the assignment of licensed radio frequency to the outdoor macrocell users, the access priority of MUEs should be higher than FUEs. In addition, the quality of service (QoS) of MUEs that is expressed in the target signal-to-interference-plus-noise ratio (SINR) must always be achieved. On the other hand, we consider an efficient QoS provisioning cost function for the low-tier FUEs. The proposed algorithm requires only local information and converges even in cases where the frontiers of available power serve the target SINRs impossible. The advantage of the algorithm is the ability to implement in a distributed manner. Simulation results show that the proposed algorithm based on our cost function provides effective resource allocation and substantial power saving as compared to the traditional algorithms.
\end{abstract}

Keywords: Femtocell; macrocell; power control; utility function; game theory

This research work is supported by the Fundamental Research Grant Scheme (FRGS /1/2012/TK06/UPNM/01/1). 


\section{Introduction}

Recent studies on wireless usage found that the most of voice calls and data traffic are originated from indoor [1]. To meet the excess demand in the wireless indoor transmission and services, femtocell has been proposed as a promising technology to increase the coverage and capacity of the network. Femtocell or a home base station (HBS) is a low-range, low-power, low-cost, and consumer owned device that is installed inside the houses and offices. HBS connects to the internet via an IP backhaul, such as digital subscriber line (DSL), cable, or WiMAX. There are two approaches for spectrum allocation between the macrocell and femtocell users: (i) spectrum splitting and (ii) spectrum sharing. In a two-tier femtocell network, the spectrum sharing approach is commonly used due to the scarcity of available spectrum and the absence of coordination between macrocell and femtocell as well as between femtocells [2]. Since femtocells operate in the licensed spectrum owned by the macrocell network, it is essential to decrease the cross-tier interference from FUEs [3]. The radio frequency (RF) interference will arise from femtocell to femtocell interference, femtocell to macrocell interference, and macrocell to femtocell interference. The femtocell to femtocell is quite small due to low transmit power and penetration losses. The near-far effect due to uneven distribution of received power is the main contributor for femtocell to macrocell interference and macrocell to femtocell interference [1]. Macrocell networks, such as CDMA networks (without existing of femtocells) employ an efficient power control to compensate for path loss, shadowing, and fading, to provide uniform coverage. Macrocell users at a cell edge need to use maximum power in the uplink transmitting, which causes unacceptable interference to nearby femtocells. Therefore, femtocells located at the cell edge experience significantly higher interference than interior femtocells. On the other hand, macrocell users at cell edge will be disrupted by femtocell transmissions since they suffer higher path loss than interior macrocell users. The problem of spectrum sharing in the two-tier femtocell networks has become a technical challenge to scientists and researchers. The implementation of distributed interference management is the main challenge in femtocell networks due to limited capacity of the signaling wire-line network (e.g., DSL links) and difference access priority between MUEs and FUEs [4, 5]. The existence of indoor femtocells make power control create a dead zones, leading to nonuniform coverage. To mitigate cross-tier interference and guarantee QoS for both MUEs and FUEs, an efficient distributed power control is necessary in both systems. Closed loop power control is commonly used in wireless networks, which consists of two algorithms loop: (i) an outer loop algorithm that updates the threshold signal-to-interference-noise-ratio (SINR) every $10 \mathrm{~ms}$ and (ii) an inner loop algorithm which computes the required powers based on the SINR measurements and it is updated every 1.25 ms. The outer loop algorithm determines the target SINR based on the estimate of the frame error rate (FER). On the other hand, the inner loop algorithm generates a power control bit based on the difference between the actual and target SINR and it sends the command to the mobile via transmit power control (TPC) [6]. For multi-hop wireless networks, multicast routing algorithms are another techniques that are used to reduce power consumption of the network [7].

Several literatures are found on the distributed power control in cellular and wireless data networks. Simple and most popular schemes of power control are proposed in [8, 9], in which all users converge to the Pareto-optimal solution whenever they can achieve the required QoS that referred to SINRs. However, QoS that referred to SINR is no longer appropriate in 
wireless data networks. Therefore, the concepts of microeconomics and game theory have been used recently to define the users QoS in terms of utility (cost) function rather than SINR and several power control schemes have been investigated $[10,11]$. The output of the game in most cases is the Nash Equilibrium of the power control game. Moreover, pricing techniques are introduced to improve the Pareto efficiency of Nash Equilibrium by an effect on the data user to maximizing the net utility [6] and [12-14]. As well as, game theoretic approach has been considered to solve the problem of interference in cognitive radio networks as [15-18]. In cognitive radio networks, unlicensed users (cognitive users) are enabled to adaptively access the frequency channels, considering the current state of the external radio environment [19]. Unlicensed users (cognitive users) can only share the unused part of the spectrum owned by the licensed users. The interference of cognitive radios should be ineffective to the QoS of the licensed users, and not exceeding the interference temperature level. Opportunistic spectrum access in cognitive radio networks has been also studied using game theory in [20], and [21]. In the context of femtocell networks, several works have been considered to mitigate the cross-tier interference [2, 22], and [23, 24]. Moreover, the game theory-based power control has been considered for HetNet small cell networks [25]. The authors addressed the mitigation of cross-tier interference problem by ensuring the protection of the macrocell users.

The main differences between the femtocell networks and traditional wireless networks are the infrastructures of the system and the different classes of users. Femtocells are low-rang (not identical with macrocells), therefore, all FUEs have a higher channel gain and require different power control algorithm. In this paper, we present a new power control scheme for the distributed interference management in two-tier femtocell networks. The objective of this algorithm is to ensure that higher priority users (MUEs) able to achieve their required QoS, whereas lower priority users demand certain QoS requirements. The main difference between this work and previous works in the traditional CDMA wireless networks is the differentiated classes of users, in which each class of users needs a different power control algorithm based on the access priority. Moreover, our work differs from previous work [22] in several aspects. Firstly, in the representation of the utility function of MUEs, the study in [22] uses sigmoid function to guarantee the minimum required SINRs. Power control algorithm using a sigmoid function is more complex because the power update formula of MUE depends on the value of optimal target SINR that need to be computed at each iteration. Instead, we defined utility function of MUEs as a square function of SINR error, and the deduced power update formula depends on the target SINR. Secondly, we introduced a new local gain in our FUEs cost function based on the local information, which can improve the performance of FUEs. The optimality of the proposed game solution is not considered due to the limitation of this work, i.e., for too heavily loaded, the proposed algorithm for MUEs and FUEs yield unacceptably low SINRs. Mobiles whose SINRs fall below a minimum QoS threshold should be dropped, otherwise, they will cause unnecessary interference to other users using the same frequency channel. The advantages of this algorithm are the ability to implement distributively, mitigate the cross-tier interference, and reduce the drain power of users. Hence, the contributions of this paper are summarized as follows:

- This paper formulates the game model based on a cost function, in which the MUEs guarantee their required QoS, while FUEs request soft QoS requirement.

- The proposed FUEs cost function contains of linear pricing function and local gain term in which it has been applied inside the utility part of the cost function. This mechanism ensures that, the transmit power of FUEs, which is included inside the SINR has been gained using local information. 
- We obtain the Nash Equilibrium of the proposed game, present the iterative power control formulas, and prove the convergence of the algorithm.

- With simulation, we show the effectiveness of the proposed power control algorithm in terms of resource allocation and power saving for different cases of the system load.

The rest of this paper is organized as follows. In Section 2, the system model of two-tier femtocell network is given, distributed interference-management algorithms are proposed and the corresponding analysis is presented in Section 3. The performance of the proposed scheme is presented by the numerical results in Section 4. Finally, we conclude this study in Section 5.

\section{System Model}

Research in traffic classification, which avoids payload inspection, has accelerated over the last five years. In this paper, we consider a typical two-tier femtocell network where $N$ femtocells are overlaid with a macrocell. Specifically, we consider the scenario where a macrocell serving $M$ macrocell user equipment (MUEs) overlaid $N$ randomly distributed femtocells. The MUEs are distributed randomly inside the coverage area of macrocell BS with radius $R_{c}$, the FUEs are randomly distributed inside the coverage area of home BS with radius $R_{f}$, and all femtocells are distributed randomly inside the coverage area of macrocell. Due to the small radius of femtocells $R_{f}$, the effect of interference between the users inside a single cell is inactive. Therefore, for simplicity, we assume that each femtocell only serves one FUE. We assume that all MUEs and FUEs are stationary so the path gains are fixed during the run time of power control simulation. We denote $C_{m}$ and $C_{f}$ as the set of MUEs and FUEs, respectively, and $C$ as the set of all users. A simple sketch of the system model of a two-tier femtocell network is shown in Fig. 1, which contains one macrocell overlaid with four femtocells. We consider the uplink scenario in this work, and $i \in C$ is referred to the $i$ th user. Let $p_{i}$ be the transmit power of user $i$ in Watt, $g_{i i}$ is the channel gain from user $i$ to its receiver with including the processing gain of the system, and $g_{i j}$ from user $j$ to the receiver of user $i \neq j$. The channel gain $g_{i j}=1 / d_{i j}^{\beta}$ with neglected shadowing and fast fading effects, where $d_{i j}$ is the distance from user $j$ to the receiver of user $i$, and $\beta$ is the path loss factor that is usually between 2 and 6 . We denote the power of additive white Gaussian noise (AWGN) at the receiver by $\sigma^{2}$ Watt. Then, the SINR obtained by user $i \in C$ at its base station can be written as

$$
\gamma_{i}=\frac{g_{i i} p_{i}}{\sum_{j \neq i} g_{i j} p_{j}+\sigma^{2}}=\frac{g_{i i} p_{i}}{I_{i}} \quad \forall i \in C
$$

where $I_{i}=\sum_{j \neq i} g_{i j} p_{j}+\sigma^{2}$ represents the aggregated interference from all FUEs and MUEs except user $i$. 


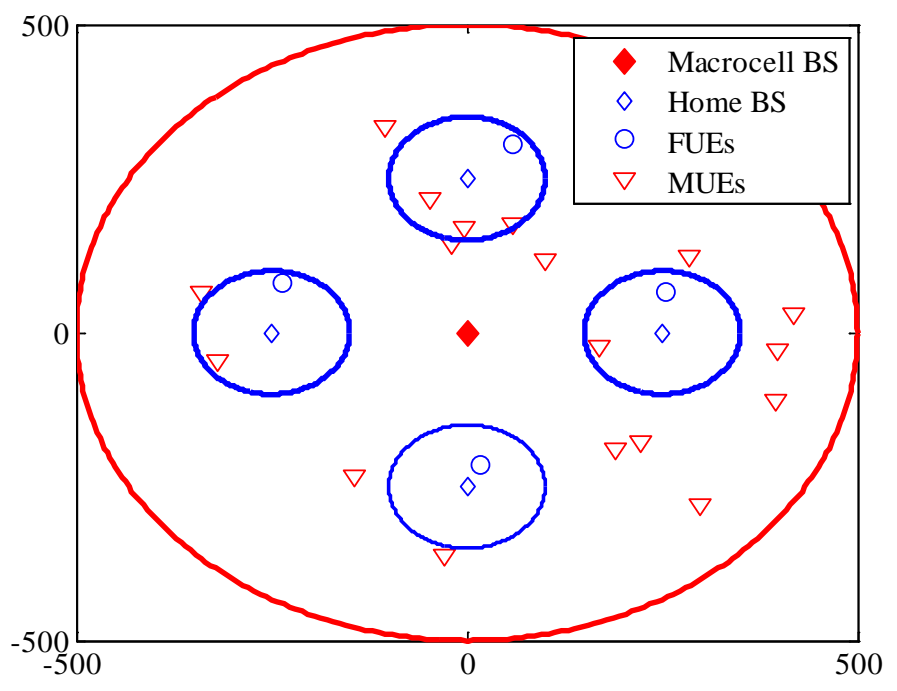

Fig. 1. System model.

To ensure adequate QoS of the higher access priority MUEs, the power control design must ensure that no MUE's SINR $\gamma_{i}$ falls below the target value $\Gamma_{i}^{m}$. Thus, there is

$$
\gamma_{i} \geq \Gamma_{i}^{m} \quad \forall i \in C_{m}
$$

On the other hand, the design of power control should ensure that the indoor lower access priority FUEs can achieve their required QoS and each $i \in C_{f}$ can attain its SINR, that is, more than a predefined threshold $\gamma_{i}^{f}$. Therefore, each FUE $i \in C_{f}$ must have that

$$
\gamma_{i} \geq \underline{\gamma_{i}^{f}} \quad \forall i \in C_{f}
$$

In this paper, we employ a cost function $J_{i}$ as a difference between the utility function of the user and its pricing function as in [11],

$$
J_{i}\left(p_{i}, \gamma_{i}(p)\right)=C_{i}\left(p_{i}\right)-U_{i}\left(\gamma_{i}(p)\right)
$$

where the power vector is $p:=\left[p_{1}, p_{2}, \ldots ., p_{N+M}\right]^{T}$. The pricing function $C_{i}\left(p_{i}\right)$ represents the cost incurred by user $i \in C$, while the utility function $U_{i}\left(\gamma_{i}(p)\right)$ represents the degree of satisfaction to the service quality. The Nash Equilibrium is the power vectors $p^{*}$ that no user can improve its cost function individually by deviating from $p_{i}^{*}$. Thus, there is

$$
J_{i}\left(p_{i}^{*}, \gamma_{i}\left(p_{i}^{*}\right)\right) \leq J_{i}\left(p_{i}^{*}, \gamma_{i}\left(p_{1}^{*}, p_{2}^{*}, \ldots ., p_{i-1}^{*}, p_{i}, p_{i+1}^{*}, \ldots, p_{N+M}^{*}\right)\right) \quad \forall i \in C
$$

The Nash Equilibrium of (4) can be obtained by taking the derivative of $J_{i}\left(p_{i}, \gamma_{i}(p)\right)$ with respect to $p_{i}$ and equating to zero as follows

$$
\frac{\partial J_{i}}{\partial p_{i}}=\frac{\partial C_{i}}{\partial p_{i}}-\frac{\partial U_{i}}{\partial \gamma_{i}} \frac{\partial \gamma_{i}}{\partial p_{i}}=0
$$

Noting that $\partial \gamma_{i} / \partial p_{i}=g_{i i} / I_{i}$, we have 


$$
U_{i}^{\prime}\left(\gamma_{i}\right)=\frac{I_{i}}{g_{i i}} C_{i}^{\prime}\left(p_{i}\right)=\frac{p_{i}}{\gamma_{i}} C_{i}^{\prime}\left(p_{i}\right)
$$

In this paper, we also further introduce a user-specific notation $\theta_{i}$ as a ratio of interference to the path gain of the user $i \in C$ as in [12] and as shown in the following

$$
\theta_{i}=\frac{I_{i}}{g_{i i}}=\frac{p_{i}}{\gamma_{i}}
$$

According to this analysis, we will explain the criteria of how to select the suitable functions $C_{i}\left(p_{i}\right)$ and $U_{i}\left(\gamma_{i}\right)$ with appropriate parameters, to design the efficient distributed power control algorithm for both MUEs and FUEs. The main goal of our algorithm is to strictly guarantee the QoS of MUEs, and we allowed slightly reduction in the QoS of FUEs in order to reduce the power consumed by the users and to mitigate the cross-tier interference.

\section{Distributed Power Control Algorithm}

\subsection{Macrocell users cost function}

The appropriate method to guarantee the QoS of MUEs is the balancing power control method, in which all MUEs achieve the same target SINR. The aim is to guarantee the QoS of higher priority MUEs by ensuring that all MUEs can meet the target SINR. On the other hand, MUEs do not need to use high power in their transmission to attain high SINR (greater than target) in order to preserve their battery life and minimize the cross-tier interference. In this case, all MUEs should have a zero price and the optimal $\gamma_{i}$ should be equal to the target SINR. For accurate communication at non-zero levels of SINR, we defined the cost of the difference between the actual SINR and the target SINR that is chosen based on the estimated FER [6]. In addition, the cost function of MUEs should be convex and positive. We thus consider the following utility and pricing functions for MUEs $i \in C_{m}$

$$
\begin{gathered}
U_{i}\left(\gamma_{i}\right)=-\left(\Gamma_{i}^{m}-\gamma_{i}\right)^{2} \\
C_{i}\left(p_{i}\right)=0
\end{gathered}
$$

Thus, according to (4), the cost function of the ith MUE can be written as

$$
J_{i}^{m}\left(p_{i}, \gamma_{i}\right)=\left(\Gamma_{i}^{m}-\gamma_{i}\right)^{2} \quad \forall i \in C_{m}
$$

The optimal $\gamma_{i}$ for each user $i \in C_{m}$ is the target SINR, which can be obtained by taking the first derivative of the MUEs cost function with respect to $\gamma_{i}$ and equating to zero,

$$
\frac{\partial J_{i}^{m}}{\partial \gamma_{i}}=0=2 \gamma_{i}-2 \Gamma_{i}^{m}
$$

Then,

$$
\hat{\gamma}_{i}=\Gamma_{i}^{m}
$$

Based on $\hat{\gamma}_{i}$ in (13), the optimal power can be obtained from (1) as $\hat{p}_{i} g_{i i} / I_{i}=\Gamma_{i}^{m}$ and the following iterative power rule can be applied 


$$
p_{i}^{(k+1)}=\frac{I_{i}^{(k)}}{g_{i i}^{(k)}} \Gamma_{i}^{m}=\frac{p_{i}^{(k)}}{\gamma_{i}^{(k)}} \Gamma_{i}^{m}
$$

where $\gamma_{i}^{(k)}$ and $p_{i}^{(k+1)}$ are the actual SINR and power of user $i$ at iteration $k$ and $k+1$, respectively. For simplicity, we can use (8) to rewrite (14). Thus,

$$
p_{i}^{(k+1)}=\theta_{i}^{(k)} \Gamma_{i}^{m}
$$

\subsection{Femtocell users cost function}

In the case of lower access priority, we assume that for each FUE, $i \in C_{f}$ also require maintaining its QoS by achieving the target SINR $\Gamma_{i}$. The target $\Gamma_{i}$ here is different from the threshold value $\gamma_{i}^{f}$ defined in (3), which in practice $\Gamma_{i}>\gamma_{i}^{f}$. The value of the target SINR should be sufficient to guarantee better service for FUEs and also should be not more high because it requires high transmit power to achieve. To decrease the cross-tier interference induced to the macrocell, FUEs should achieve its target SINR using the minimum required transmit power. In game theory, the selection of a cost function is an important problem because it is a basis of the game, which will deduce the power iterative algorithm. FUE has two conflicting objectives: (i) achieve better service by obtaining higher SINR and (ii) higher SINR is achieved at the cost of an increased drain on the battery and higher cross-interference to others FUEs and MUEs. Therefore, the cost function for each FUE should be depended on power and SINR, and it should be non-negative and convex to allow the existence of a non-negative minimum. In addition, the target $\Gamma_{i}$ should be included inside the cost function to be varied according to the service requirement. Higher $\Gamma_{i}$ can be chosen for voice users and lower target can be chosen for data users. Thus, we consider the cost function of the ith FUE as in [6] with a new special parameter as

$$
J_{i}^{f}\left(p_{i}, \gamma_{i}\left(p_{i}\right)\right)=b_{i} p_{i}+\left(\Gamma_{i}-e^{a_{i} \theta_{i}} \gamma_{i}\right)^{2} \quad \forall i \in C_{f}
$$

where $a_{i}$ and $b_{i}$ are non-negative weighting factors. It is shown from (16) that the linear pricing power $b_{i} p_{i}$ is always positive, and the square of SINR error $\left(\Gamma_{i}-e^{a_{i} \theta_{i}} \gamma_{i}\right)^{2}$ is always positive due to the square function. In addition, the proper selection of the non-negative weighting factors in the cost function equation (16) is important. Choosing $b_{i} /\left(e^{a_{i} \theta_{i}}\right)^{2}>1$ places more emphasis on power usage, whereas $b_{i} /\left(e^{a_{i} \theta_{i}}\right)^{2}<1$ places more emphasis on the SINR.

The new special local gain term $e^{a_{i} \theta_{i}}$ is the advantage of our proposed algorithm, in which it can guide FUEs to an efficient Nash Equilibrium point when the system operates in different loads. We defined $e^{a_{i} \theta_{i}}$ as a local gain term because it only depends on the weighting factor $a_{i}$ and a user-specific notation $\theta_{i}$ (a ratio of FUEs power and SINR). That means, the local gain term only depends on local information and it does not need any other information from home BS. The utility part of the cost function (16) will guide all FUEs to achieve the target SINR, but the Nash Equilibrium may actually less than the target SINR due to the pricing term and the new special gain parameter. Nevertheless, slight decrease in FUEs SINR resulting in substantial reduction in transmitting power as well as significant reduction in the cross-tier interference. Now, applying the necessary condition of Nash Equilibrium to the $i$ th FUEs cost 
function yields:

$$
\begin{aligned}
\frac{\partial J_{i}^{f}}{\partial p_{i}}=0 & =b_{i}-2 e^{a_{i} \theta_{i}}\left(\Gamma_{i}-e^{a_{i} \theta_{i}} \gamma_{i}\right) \frac{\partial \gamma_{i}}{\partial p_{i}} \\
& =b_{i}-2 e^{a_{i} \theta_{i}}\left(\Gamma_{i}-e^{a_{i} \theta_{i}} \gamma_{i}\right) \frac{g_{i i}}{I_{i}}
\end{aligned}
$$

Rearranging terms of (18) yields

$$
\gamma_{i}=\frac{\Gamma_{i}}{e^{a_{i} \theta_{i}}}-\frac{b_{i} I_{i}}{2 g_{i i}\left(e^{a_{i} \theta_{i}}\right)^{2}}
$$

It follows from (19) that as $b_{i} \rightarrow 0$, the power expenditure increases and the SINR $\gamma_{i} \rightarrow \Gamma_{i} / e^{a_{i} \theta_{i}}$. On the other hand, as $a_{i} \rightarrow 0$ and $b_{i} \rightarrow 0$, the SINR will be converging to the target $\gamma_{i} \rightarrow \Gamma_{i}$. Substituting for $\gamma_{i}$ from (1) and isolating $p_{i}$, we can obtain the power in terms of given and measured quantities as

$$
p_{i}=\frac{\Gamma_{i}}{e^{a_{i} \theta_{i}}} \frac{I_{i}}{g_{i i}}-\frac{b_{i} I_{i}^{2}}{2 g_{i i}^{2}\left(e^{a_{i} \theta_{i}}\right)^{2}}
$$

At Nash Equilibrium, the power value can thus be computed as

$$
p_{i}^{*}=\max \left(\frac{\Gamma_{i}}{e^{a_{i} \theta_{i}}} \frac{I_{i}}{g_{i i}}-\frac{b_{i} I_{i}^{2}}{2 g_{i i}^{2}\left(e^{a_{i} \theta_{i}}\right)^{2}}, 0\right)
$$

To present (20) as a numerical algorithm, we assume that the algorithm will run in real time with potential measurements updated every step of the algorithm [6]. Thus, the iterative power rule can be written as

$$
p_{i}^{(k+1)}=\frac{\Gamma_{i}}{e^{a_{i} \theta_{i}^{(k)}}} \frac{I_{i}^{(k)}}{g_{i i}^{(k)}}-\frac{b_{i}\left(I_{i}^{(k)}\right)^{2}}{2\left(g_{i i}^{(k)}\right)^{2}\left(e^{a_{i} \theta_{i}^{(k)}}\right)^{2}}
$$

We define $p_{i}^{(k+1)}=f_{i}^{(k)} p_{i}^{(k)}$ and based on (14), we can get

$$
f_{i}^{(k)} p_{i}^{(k)}=p_{i}^{(k+1)}=\frac{\Gamma_{i}}{e^{a_{i} \theta_{i}^{(k)}}} \frac{I_{i}^{(k)}}{g_{i i}^{(k)}}-\frac{b_{i}\left(I_{i}^{(k)}\right)^{2}}{2\left(g_{i i}^{(k)}\right)^{2}\left(e^{a_{i} \theta_{i}^{(k)}}\right)^{2}}, \quad p_{i}^{(k)}>0
$$

where $\theta_{i}^{(k)}=p_{i}^{(k)} / \gamma_{i}^{(k)}$ as in (8). The initial condition associated with (23) must satisfy $p_{i}^{(0)} \neq 0$. Note that, the positive term in the expression of (23) is different to the power balancing solution, in which the new specific parameter $e^{a_{i} \theta_{i}}$ has been added to the denominator. As well as, the negative term is proportional to the square of interference, and the square of exponential of interference. The two formulas of algorithm (22) and (23) require only a single measurement at each step of the iteration, so the power control can be used to implement as a distributed power control. 


\subsection{Convergence}

In [26], the authors show that if a fixed point of the algorithm $p^{(k+1)}=f\left(p^{(k)}\right)$ exist and the function $f$ satisfy the following three conditions:

1) Positivity $f(p) \geq 0$,

2) Monotonicity $p \geq p^{\prime} \Rightarrow f(p) \geq f\left(p^{\prime}\right)$,

3) Scalability $\forall \alpha \geq 1$; $\alpha f(p) \geq f(\alpha p)$.

then the algorithm converges to a fixed and unique point.

For the MUEs, the above three properties are obviously satisfied as has been explained in [25].

On the other hand, from (22), and in terms of interference, the positivity requires

$$
I_{i}=-\frac{g_{i i} \text { LambertW }\left(-\frac{2 a_{i} \Gamma_{i}}{b_{i}}\right)}{a_{i}}
$$

where LambertW(.) is the LambertW function. When we selected a proper value of $a_{i} / b_{i}$, the value of LambertW $\left(-2 a_{i} \Gamma_{i} / b_{i}\right)$ will be small positive quantity and the positive condition can be easily met.

For monotonicity, it is enough to have an increasing best response function respect to interference $I_{i}$. Thus, if we differentiate (22) with respect to $I_{i}$, we get

$$
\frac{\partial f(p)}{\partial\left(I_{i}\right)}=\frac{a_{i} b_{i}\left(I_{i}\right)^{2}}{\left(g_{i i}\right)^{3}\left(e^{\frac{a_{i} I_{i}}{g_{i i}}}\right)^{2}}-\frac{a_{i} b_{i}}{\left(g_{i i}\right)^{2}\left(e^{\frac{a_{i} I_{i}}{g_{i i}}}\right)^{2}}+\frac{\Gamma_{i}}{g_{i i} e^{\frac{a_{i} I_{i}}{g_{i i}}}}-\frac{a_{i} I_{i} \Gamma_{i}}{\left(g_{i i}\right)^{2} e^{\frac{a_{i} I_{i}}{g_{i i}}}}
$$

Using (25), for monotonicity, we should have

$$
\Gamma_{i}>\frac{b_{i} I_{i}}{g_{i i} e^{\frac{a_{i} I_{i}}{g_{i i}}}} \Rightarrow I_{i}<-\frac{g_{i i} \text { LambertW }\left(-\frac{a_{i} \Gamma_{i}}{b_{i}}\right)}{a_{i}}
$$

which is stricter than (24). Finally, the condition of the scalability in our method can be written as 


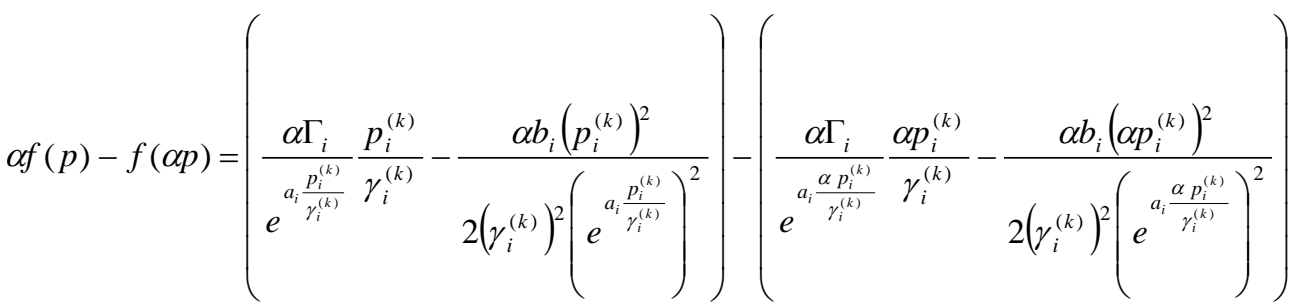

$$
\begin{aligned}
& =\Gamma_{i}\left(\frac{1}{e^{a_{i} p_{i}^{(k)}}}-\frac{1}{\gamma_{i}^{(k)}} e^{a_{i} \frac{\alpha p_{i}^{(k)}}{\gamma_{i}^{(k)}}}\right)-\frac{b_{i} p_{i}^{(k)}}{2 \gamma_{i}^{(k)}}\left(\frac{1}{e^{2 a_{i} p_{i}^{(k)}}}-\frac{\alpha}{e^{2 a_{i} \frac{\alpha p_{i}^{(k)}}{\gamma_{i}^{(k)}}}}\right)
\end{aligned}
$$

So, for scalability, it is enough that positivity be met.

\section{Simulation Results}

In this section, we present the numerical results of our proposed power control algorithm and compare the performance with the traditional algorithm without local gain. The traditional algorithm has been proposed in [6], and it is applied in femtocell network in [22], which can be shown in the following equation:

$$
p_{i}^{(k+1)}=\Gamma_{i} \frac{I_{i}^{(k)}}{g_{i i}^{(k)}}-\frac{b_{i}\left(I_{i}^{(k)}\right)^{2}}{2 c_{i}\left(g_{i i}^{(k)}\right)^{2}}
$$

In this simulation, we compared our proposed algorithm with the traditional algorithm [22] respecting to femtocell users only, while the power update formula of macrocell users is different. To perform a fair comparison with the traditional algorithm, we kept the same physical parameters for all network elements, such as MUEs, FUEs, and base stations. The local gain proposed in our FUEs cost function has been appearing in both positive and negative terms in (22). One of the advantages of our proposed algorithm can be found in the positive term of (22), in which the algorithm guides FUEs to achieve $\Gamma_{i} / e^{a_{i} \theta_{i}}$ rather than the target $\Gamma_{i}$ that was achieved by the traditional algorithm [22]. On the other hand, the reduction of power is also affected by the local gain $e^{a_{i} \theta_{i}}$ that appears in the denominator of the negative term of (22).

The network settings and the deployment of users in this simulation are illustrated in Fig. 1, where the MUEs are randomly deployed inside the circle of the radii of $R_{c}=500 \mathrm{~m}$ and serving by the macro BS that located in the center. The FUEs are also randomly deployed inside small circle of the radii of $R_{f}=100 \mathrm{~m}$ and we assume that each femtocell BS serves only one FUE. The initial power of all users was $p_{i}^{(0)}=2.22 \times 10^{-16} \mathrm{~W}$ in both simulations. The path gain from the transmitter user $j \in C$ to the receiver of user $i$ is calculated as $1 / d_{i j}^{\beta}$ where $d_{i j}$ is the distance and $\beta$ is the path loss exponent. The same pricing coefficients $a_{i}$ and $b_{i}$ are used for all FUEs. The number of MUEs and FUEs, the values of SINR targets $\Gamma_{i}^{m}, \Gamma_{i}$ and the other simulation parameters are summarized in Table 1. 
Table 1. Simulation parameters.

\begin{tabular}{|c|c|}
\hline Parameter & Value \\
\hline Number of users, $M, N$ & 10,40 \\
\hline Processing Gain $G$ & 100 \\
\hline Path-loss exponent, $\beta$ & 3 \\
\hline$\Gamma_{i}^{m}, \Gamma_{i}$ & 5,4 \\
\hline Noise power $\sigma^{2}$ in Watt & $10^{-10}$ \\
\hline$a_{i}, b_{i} \forall i \in C_{f}$ & 5000,15000 \\
\hline
\end{tabular}

In this simulation, all MUEs and FUEs are distributed randomly inside the area of their own cells and each FUE or MUE has a different value of path gain. The path gain of each user depends on its distance from its own base station. The path gains of users will be computed from different positions, so the static scenario has been considered in our simulation. In multiple curves figures, a single curve corresponds to one specific user. All MUEs in both algorithms update their transmission power using (14). The admission control is not considered in this simulation; therefore, no FUE is removed from the system and the value of threshold $\underline{\gamma_{i}^{f}}$ has been neglected. The simulation executed three times: in low, medium, and high system loads, respectively, and the present figures represented the evolutions of power and SINR and their average. The increase of system load shows how the ability of macrocell users to guarantee their QoS, and also shows the SINR degradation of femtocell users. In addition, increasing system loads show a significant reduction of powers among FUEs and MUEs that cannot be seen clearly in the low load system. We noted that the curves in Fig. 2, Fig. 4, and Fig. 6, represent the values of power and SINR of all MUEs and FUEs with iterations. The curves in red colors represent the values of power and SINR of MUEs, whereas the curves in blue colors represent the values of power and SINR of FUEs. It is easy to see in the following simulation that all MUEs in red curves consume higher power than FUEs in the blue curves, because FUEs are low-range and they have higher channel gain than MUEs. On the other hand, it is shown that the SINR values of all higher priority MUEs are the same (all MUEs converge to the target SINR $\Gamma_{i}^{m}=5$ ) without any reduction. The reason is due to the successful choice of MUEs utility function with zero-pricing function. The linear pricing function that applied to the FUEs cost function is the reason of the reduction in the values of FUEs SINR, as shown in the blue curves of medium and high load system.

In Fig. 2, we display the evolutions of power and SINR in both algorithms for all MUEs and FUEs in the low load system. All MUEs and FUEs converge to the SINR requirements at the equilibrium, but the power consumed by MUEs and FUEs in the proposed algorithm is less than the power consumed by users in the traditional algorithm. The reduction in power cannot be seen clearly due to the density of user's power curves in the figure, so the average performance has been computed and displayed in the following figure. Fig. 3 presents the average power of MUEs and the average power and SINR of FUEs. We found that the average SINR are $\bar{\gamma}_{i}^{f}=3.992$, and $\bar{\gamma}_{i}^{f}=3.997$ in the proposed algorithm and traditional algorithm, respectively. On the other hand, the average power of MUEs reduced to 4.39\% (final average power $\bar{p}_{i}^{m}=2.2254 \times 10^{-5}$ versus $\bar{p}_{i}^{m}=2.3276 \times 10^{-5}$ in the proposed algorithm and traditional, respectively), and the power FUEs reduced by $4.5 \%$ (final average power $\bar{p}_{i}^{f}=1.1935 \times 10^{-6}$ versus $\bar{p}_{i}^{f}=1.2505 \times 10^{-6}$ in the proposed algorithm and traditional algorithm, 
respectively). The minimum and maximum values of SINR and power for MUEs and PUEs have been shown in Table 2.
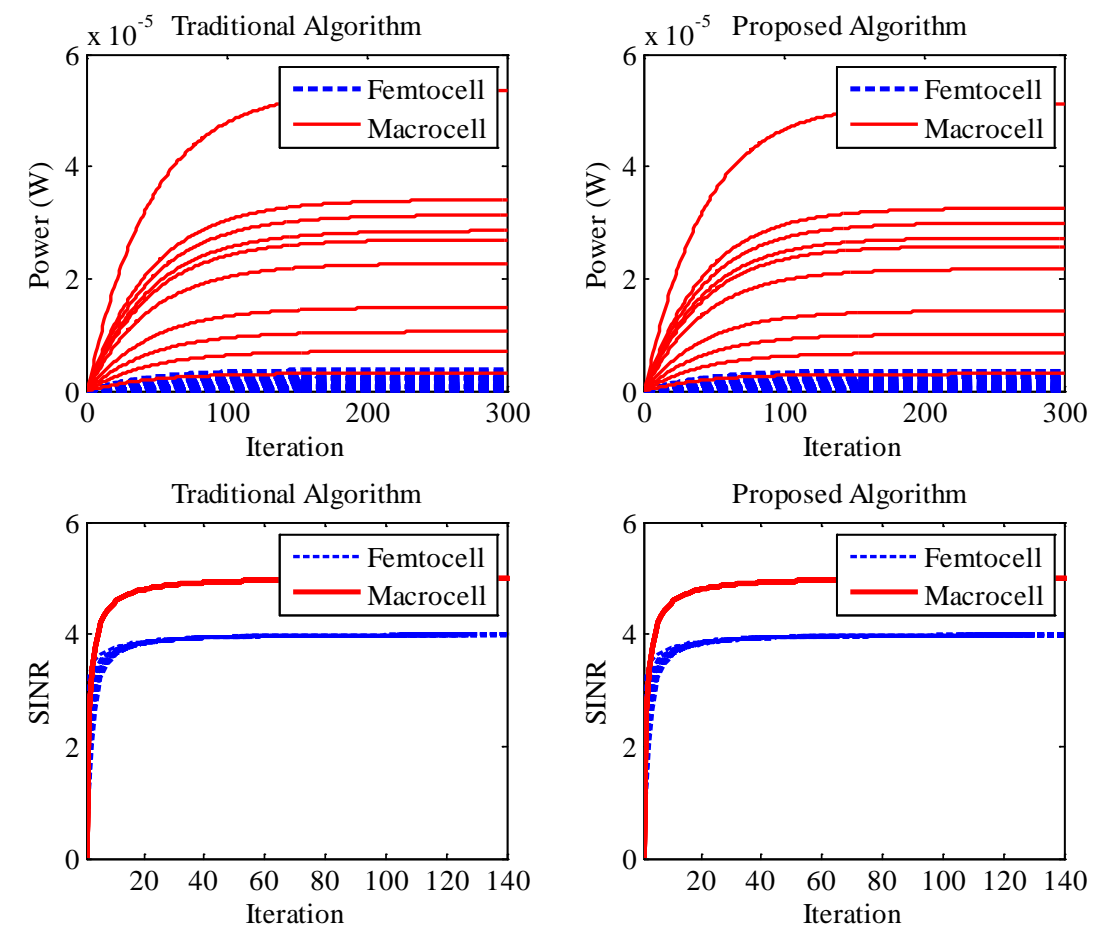

Fig. 2. Performance comparison of proposed algorithm and traditional algorithm in low load system for all MUEs and FUEs.
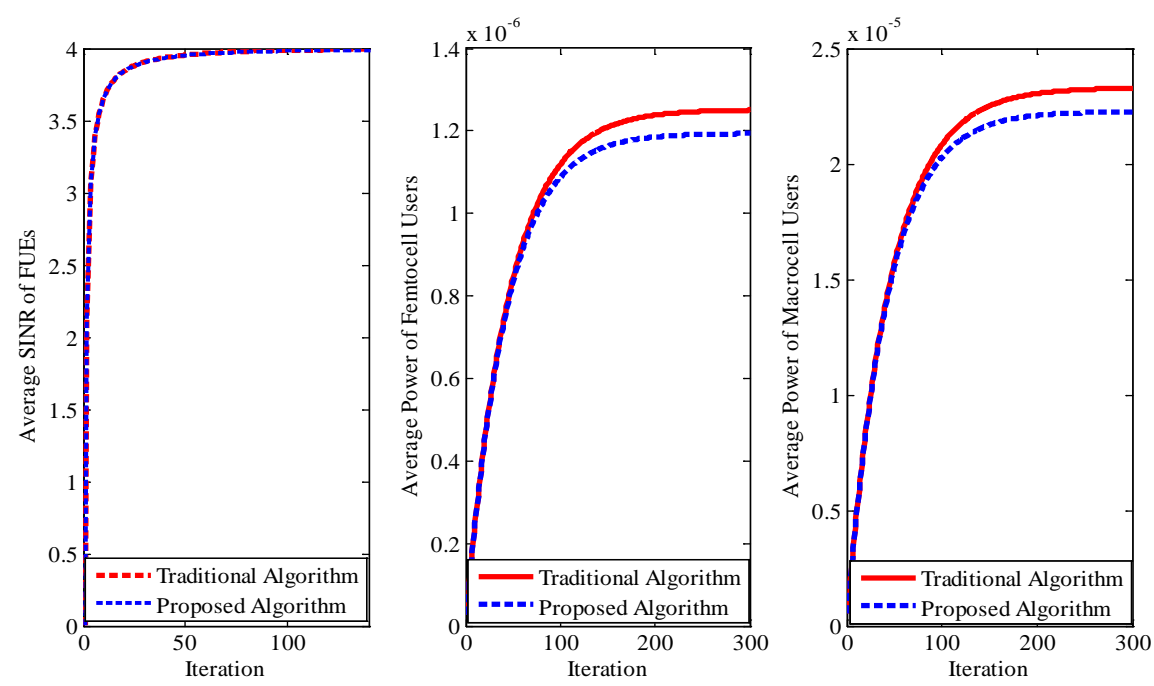

Fig. 3. Average performance comparison of proposed algorithm and traditional algorithm in low load system. 
Table 2. Min, max SINR and power evaluations.

\begin{tabular}{|c|c|c|c|c|c|c|}
\hline & \multicolumn{6}{|c|}{ Low load system } \\
\hline Values & $\begin{array}{l}\text { Min } \\
\gamma_{i}^{f}\end{array}$ & $\begin{array}{c}\operatorname{Max} \\
\gamma_{i}^{f}\end{array}$ & $\operatorname{Min} p_{i}^{f}$ & $\operatorname{Max} p_{i}^{f}$ & Min $p_{i}^{m}$ & $\operatorname{Max} p_{i}^{m}$ \\
\hline $\begin{array}{l}\text { Proposed } \\
\text { Algorithm }\end{array}$ & 3.9728 & 3.996 & $3.9351 \times 10^{-8}$ & $3.4418 \times 10^{-6}$ & $2.9145 \times 10^{-6}$ & $4.9504 \times 10^{-5}$ \\
\hline \multirow[t]{2}{*}{ Traditional } & 3.9891 & 3.996 & $4.0662 \times 10^{-8}$ & $3.5867 \times 10^{-6}$ & $3.0199 \times 10^{-6}$ & $5.1297 \times 10^{-5}$ \\
\hline & \multicolumn{6}{|c|}{ Medium load system } \\
\hline Values & $\begin{array}{l}\text { Min } \\
\gamma_{i}^{f}\end{array}$ & $\begin{array}{c}\operatorname{Max} \\
\gamma_{i}^{f}\end{array}$ & $\operatorname{Min} p_{i}^{f}$ & $\operatorname{Max} p_{i}^{f}$ & Min $p_{i}^{m}$ & $\operatorname{Max} p_{i}^{m}$ \\
\hline $\begin{array}{c}\text { Proposed } \\
\text { Algorithm }\end{array}$ & 2.7726 & 3.994 & $7.8327 \times 10^{-7}$ & $1.5699 \times 10^{-4}$ & $1.3799 \times 10^{-4}$ & 0.0038 \\
\hline \multirow[t]{2}{*}{ Traditional } & 2.6635 & 3.9946 & $2.438 \times 10^{-6}$ & $4.746 \times 10^{-4}$ & $4.3505 \times 10^{-4}$ & 0.0121 \\
\hline & \multicolumn{6}{|c|}{ High load system } \\
\hline Values & $\begin{array}{c}\text { Min } \\
\gamma_{i}^{f}\end{array}$ & $\begin{array}{c}\operatorname{Max} \\
\gamma_{i}^{f} \\
\end{array}$ & Min $p_{i}^{f}$ & $\operatorname{Max} p_{i}^{f}$ & Min $p_{i}^{m}$ & $\operatorname{Max} p_{i}^{m}$ \\
\hline $\begin{array}{l}\text { Proposed } \\
\text { Algorithm }\end{array}$ & 1.285 & 3.993 & $1.2083 \times 10^{-6}$ & $3.0408 \times 10^{-4}$ & $4.465 \times 10^{-4}$ & 0.0204 \\
\hline Traditional & 0.0546 & 3.995 & $2.59 \times 10^{-6}$ & $5.294 \times 10^{-4}$ & $9.955 \times 10^{-4}$ & 0.0456 \\
\hline
\end{tabular}

The advantage of the proposed algorithm is appeared clearly in the medium load test, as shown in Fig. 4, and Fig. 5. The average power reduction of MUEs is 68\% (final average power $\bar{p}_{i}^{m}=0.0015$ versus $\bar{p}_{i}^{m}=0.0047$ in the proposed algorithm and traditional algorithm, respectively), and the reduction average power of femtocell users is $68 \%$ (final average power $\bar{p}_{i}^{f}=4.9749 \times 10^{-5}$ versus $\bar{p}_{i}^{f}=1.5670 \times 10^{-4}$ in the proposed algorithm and traditional algorithm). On the other hand, the evaluations show that only $0.038 \%$ reduction in average SINR, $\bar{\gamma}_{i}^{f}=3.6372$ in the proposed algorithm as opposed to $\bar{\gamma}_{i}^{f}=3.6502$ in the traditional algorithm. As shown in Table 2, the minimum value of FUEs SINR of the farthest user in our proposed algorithm is higher than traditional algorithms. 

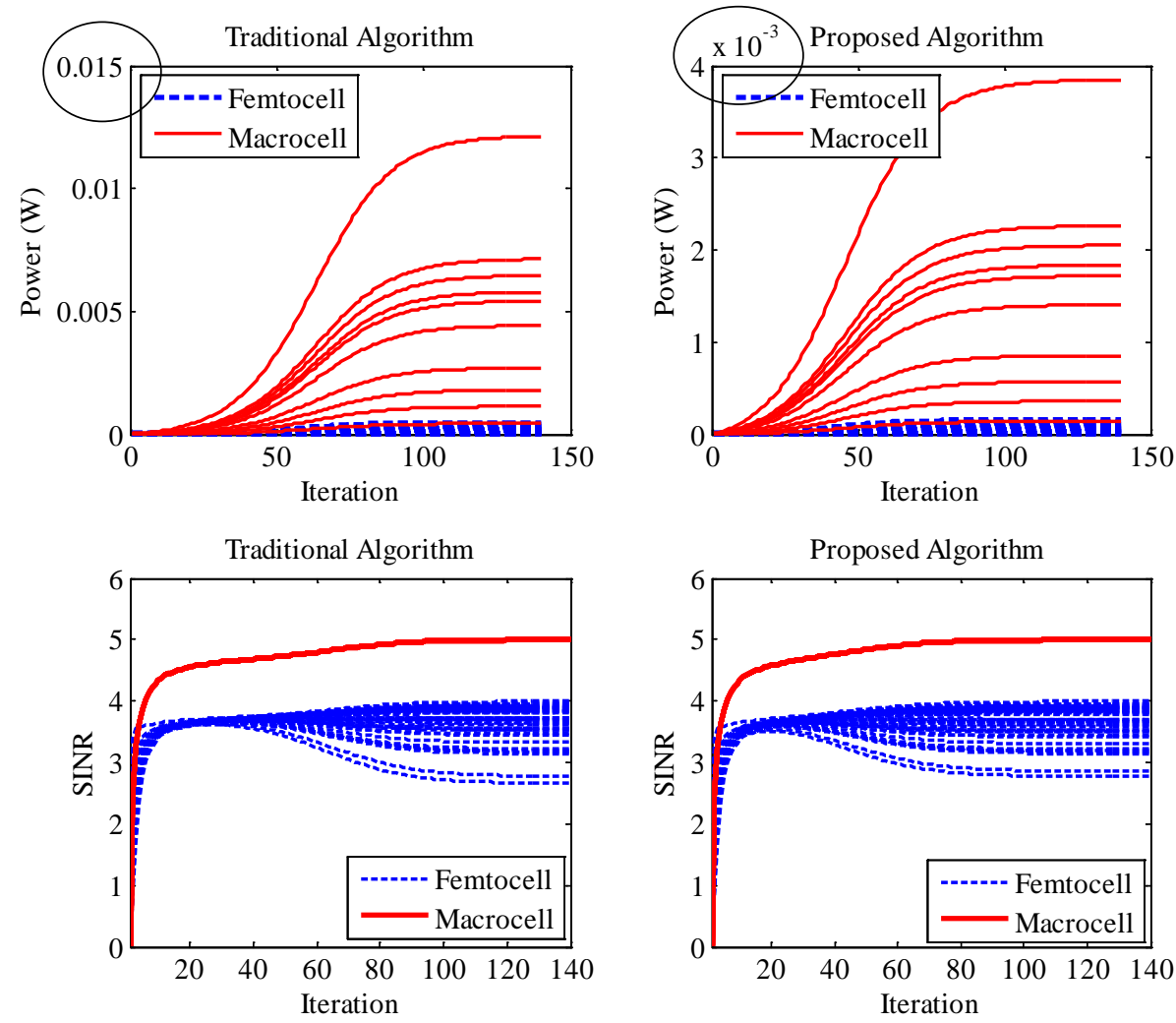

Fig. 4. Performance comparison of proposed algorithm and traditional algorithm in medium load system for all MUEs and FUEs.
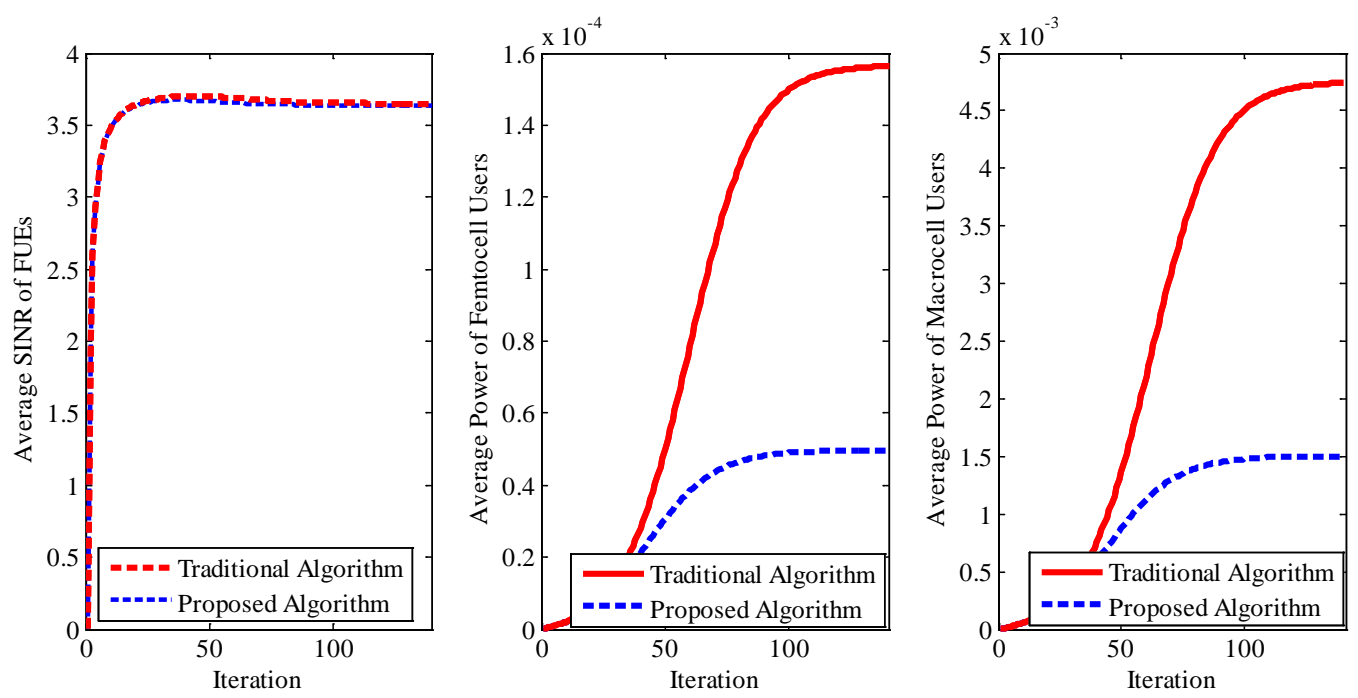

Fig. 5. Average performance comparison of proposed algorithm and Traditional algorithm in medium load system. 
In Fig. 6 and Fig. 7, we display the evaluations of power and SINR in the high load network. Both algorithms smoothly reduce the SINRs of FUEs to let MUEs to reach their desired SINR target, but the SINRs of FUEs in our proposed algorithm has reasonable values. The average value of FUEs SINR is $\bar{\gamma}_{i}^{f}=3.108$ in both algorithms. In addition, the reduction of the average power of MUEs is $55.2 \%$ (final average power $\bar{p}_{i}^{m}=0.0072$ versus 0.0161 in the proposed algorithm and traditional algorithm, respectively), and the reduction of the average power of FUEs is $55.7 \%\left(\bar{p}_{i}^{f}=1.2715 \times 10^{-4}\right.$ versus $\bar{p}_{i}^{f}=2.5061 \times 10^{-4}$ in the proposed algorithm and traditional algorithm). Furthermore, the range of FUEs SINRs in the proposed algorithm is more suitable where the min value is $\gamma_{i}^{f}=1.285$ as opposed to $\gamma_{i}^{f}=0.0546$.
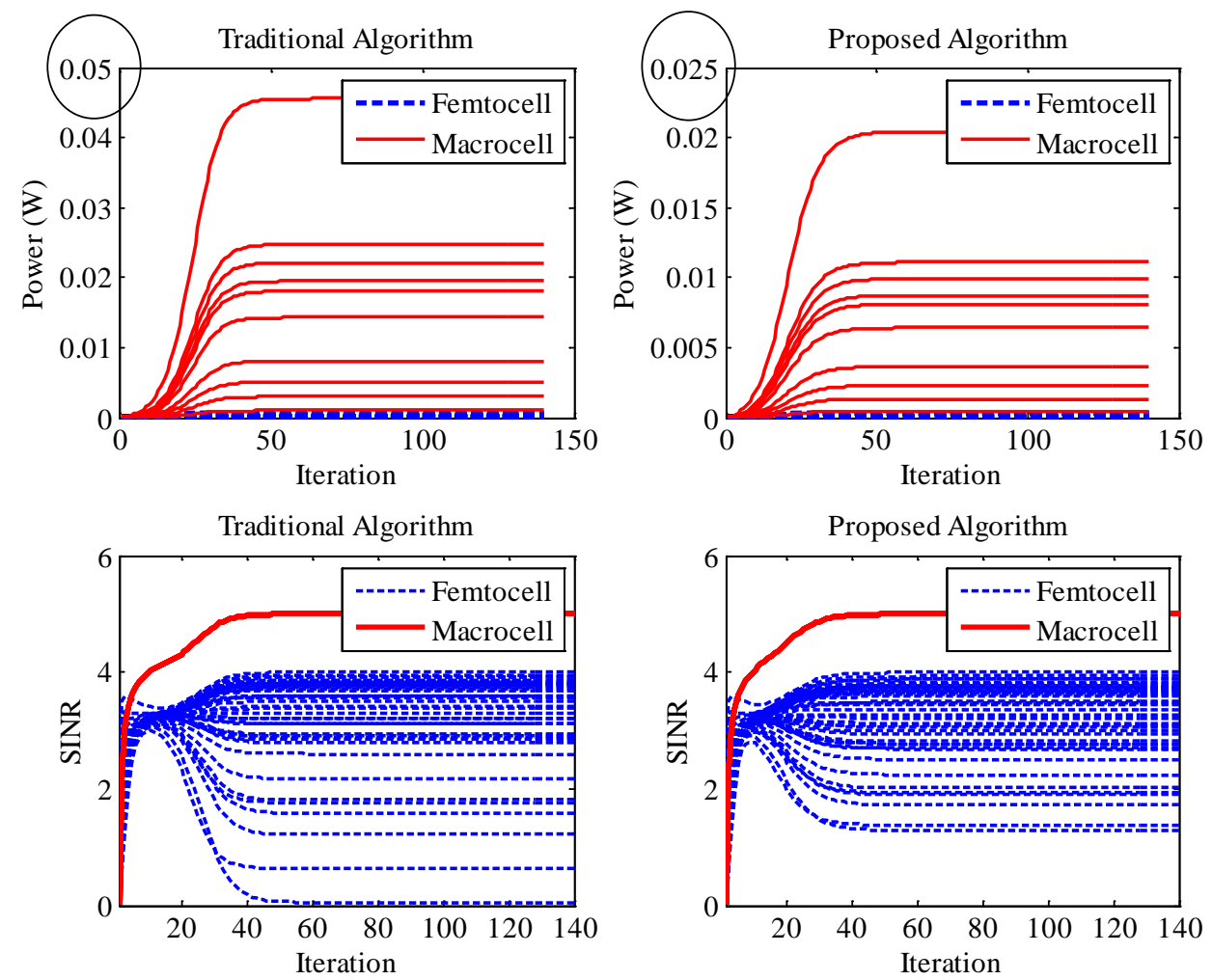

Fig. 6. Performance comparison of proposed algorithm and traditional algorithm in high load system for all MUEs and FUEs, $a_{i}=4000$. 

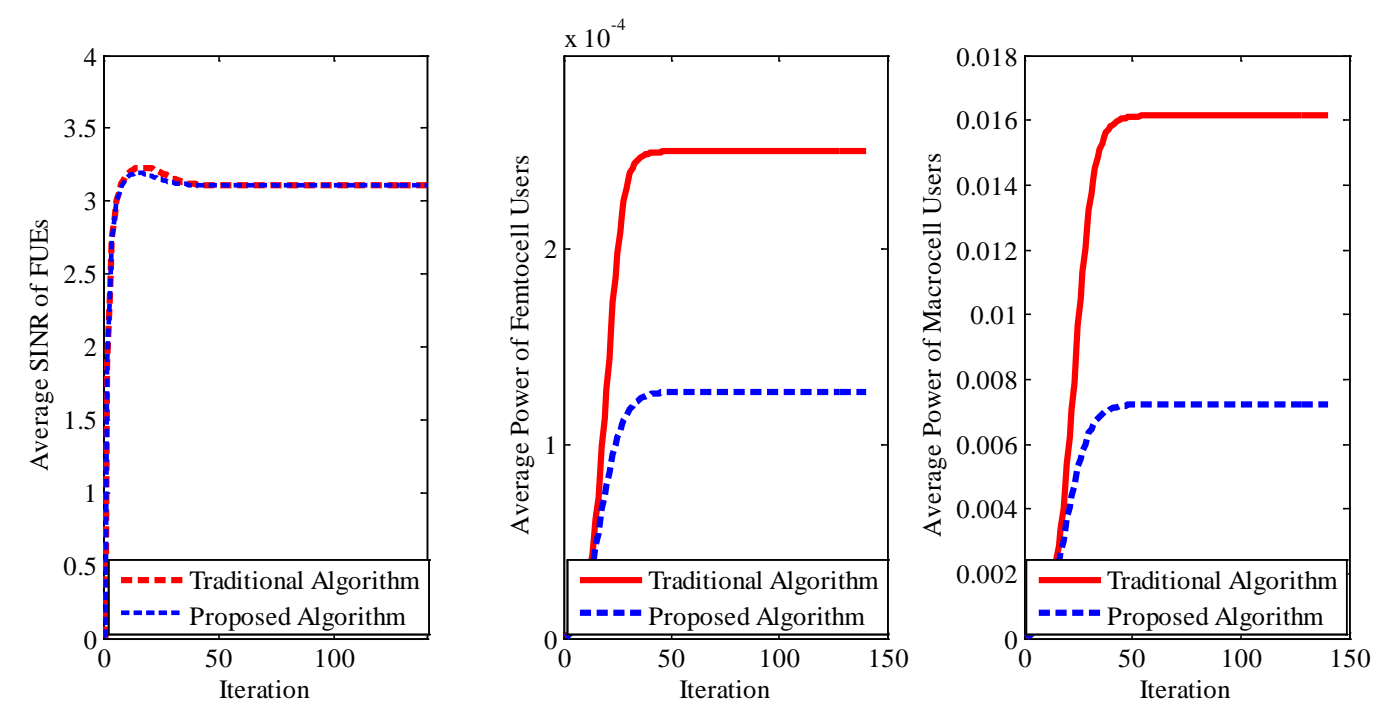

Fig. 7. Average performance comparison of proposed algorithm and traditional algorithm in high load system.

\section{Conclusion}

In this paper, we have proposed a new power control algorithm to manage the distributed interference in the two-tier networks. Specifically, a new design of power control for the FUEs has been considered. It has been shown that the proposed power control algorithm of FUEs is able to mitigate the cross-tier interference, making the MUEs maintaining their desired SINR requirements easily. The convergence of the proposed power control algorithm has been proved analytically and the features are confirmed through comparison in the numerical study. An interesting topic for future work would be the development of an admission control algorithm or link quality protection mechanism together with power control algorithm to improve the system performance further.

\section{References}

[1] V. Chandrasekhar, J. Andrews, A. Gatherer, "Femtocell networks: a survey," IEEE Communications Magazine, vol. 46, no. 9, pp. 59-67, 2008. Article (CrossRef Link)

[2] X. Kang, R. Zhang, M. Motani, "Price-based resource allocation for spectrum-sharing femtocell networks: A stackelberg game approach,” IEEE Journal on Selected areas in Communications, vol. 30, no. 3, pp. 538-549, 2012. Article (CrossRef Link)

[3] G. De La Roche, A. Valcarce, D. López-Pérez, J. Zhang, "Access control mechanisms for femtocells," IEEE Communications Magazine, vol. 48, no. 1, pp. 33-39, 2010. Article (CrossRef Link)

[4] J. Han-Shin, C. Mun, J. Moon, J. Yook, "Interference mitigation using uplink power control for two-tier femtocell networks," IEEE Transactions on Wireless Communications, vol. 8, no. 10, pp. 4906-4910, 2009. Article (CrossRef Link)

[5] I. Guvenc, J. Moo-Ryong, F. Watanabe, H. Inamura, "A hybrid frequency assignment for femtocells and coverage area analysis for co-channel operation," IEEE Communications Letters, vol. 12, no. 12, pp. 880-882, 2008. Article (CrossRef Link)

[6] S. Koskie, Z. Gajic, "A Nash game algorithm for SIR-based power control in 3G wireless CDMA 
networks,” IEEE/ACM Transactions on Networks (TON), vol. 13, no. 5, pp. 1017-1026, 2005. Article (CrossRef Link)

[7] D. Jiang, Z. Xu, W. Li, Z. Chen, “Topology control-based collaborative multicast routing algorithm with minimum energy consumption,” International Journal of Communication Systems, 2014. Article (CrossRef Link)

[8] G. Foschini and Z. Miljanic, "A simple distributed autonomous power control algorithm and its convergence,” IEEE Transactios on Vehicular Technology, vol. 42, no. 4, pp. 641-646, 1993. Article (CrossRef Link)

[9] J. Zander, "Performance of optimum transmitter power control in cellular radio systems," IEEE Transactions on Vehicular Technology, vol. 41, no. 1, pp. 57-62, 1992. Article (CrossRef Link)

[10] H. Ji, C. Huang, "Non-cooperative uplink power control in cellular radio systems,” Journal Wireless Networks, vol. 4, no. 3, pp. 233-240, 1998. Article (CrossRef Link)

[11] T. Alpcan, T. Başar, R. Srikant, E. Altman, "CDMA uplink power control as a noncooperative game,” Journal Wireless Networks, vol. 8, no. 6, pp. 659-670, 2002. Article (CrossRef Link)

[12] M. Xiao, N. Shroff and E. Chong, "Utility-based power control in cellular wireless systems," in Proc. of the Twentieth Annual Joint Conference of the IEEE Computer and Communications Societies (INFOCOM 2001), pp. 412-421, 2001. Article (CrossRef Link)

[13] Cem U. Saraydar, N. Mandayam, D. Goodman, "Pricing and power control in a multicell wireless data network," IEEE Journal on Selected areas in Communications, vol. 19, no. 10, pp. 1883-1892, 2001. Article (CrossRef Link)

[14] Cem U. Saraydar, N. Mandayam and D. Goodman, "Efficient power control via pricing in wireless data networks," IEEE Transactions on Wireless Communications, vol. 50, no. 2, pp. 291-303, 2002. Article (CrossRef Link)

[15] S. Jayaweera, T. Li, "Dynamic spectrum leasing in cognitive radio networks via primary-secondary user power control games,” IEEE Transactions on Wireless Communications, vol. 8, no. 6, pp. 3300-3310, 2009. Article (CrossRef Link)

[16] Y. A. Al-Gumaei, K. Dimyati, "Optimal power control game for primary-secondary user in cognitive radio network,” International Journal of Physical Science vol. 5, no. 4, pp. 345-351, 2010. Article (CrossRef Link)

[17] F. Li, X. Tan, L. Wang, “A new game algorithm for power control in cognitive radio networks," IEEE Transactions on Vehicular Technology, vol. 60, no. 9, pp. 4384-4391, 2011. Article (CrossRef Link)

[18] Y. A. Al-Gumaei, K. A. Noordin, A. W. Reza, K. Dimyati, “A New SIR-Based Sigmoid Power Control Game in Cognitive Radio Networks” PloS one, vol. 9, no. 10: e109077, 2014. Article (CrossRef Link)

[19] S. Hu, Y. D. Yao, Z. Yang, "MAC Protocol Identification Using Support Vector Machines for Cognitive Radio Networks,” IEEE Wireless Communications, vol. 21, no. 1, pp. 52-60, 2014. Article (CrossRef Link)

[20] Y. Xu, J. Wang, Q. Wu, A. Anpalagan, “Opportunistic spectrum access in cognitive radio networks: global optimization using local interaction games,” IEEE Journal of Selected Topics in Signal Processing, vol. 6, no. 2, pp. 180-194, 2012. Article (CrossRef Link)

[21] Y. Xu, Q. Wu, J. Wang, L. Shen, A. Anpalagan, “Opportunistic pectrum access using partially overlapping channels: Graphical game and uncoupled learning," IEEE Transactions on Communications, vol. 61, no. 9, pp. 3906-3918, 2013. Article (CrossRef Link)

[22] D. Ngo, L. Le, T. Le-Ngoc, E. Hossain, D. Kim, "Distributed interference management in two-tier CDMA femtocell networks,” IEEE Transactions on Wireless Communications, vol. 11, no. 3, pp. 979-989, 2012. Article (CrossRef Link)

[23] Q. Han, K. Ma, X. Wang, X. Guan, J. Ma, "Stackelberg game based interference management for two-tier femtocell networks,” Journal Wireless Networks, vol. 19, no. 7, pp. 1-13, Mar. 2013. Article (CrossRef Link)

[24] R. Xie, F. Richard Yu, H. Ji, "Interference management and power allocation for energy-efficient cognitive femtocell networks,” Journal Mobile Networks and Applications, vol. 18, no. 4, pp. 578-590, 2013. Article (CrossRef Link) 
[25] X. Xu, Y. Li, R. Gau, X. Tao, “Joint voronoi diagram and game theory-based power control scheme for the HetNet small cell networks,” EURASIP Journal on Wireless Communication and Networking, 2014:213, 2014. Article (CrossRef Link)

[26] R. D. Yates, “A framework for uplink power control in cellular radio systems,” IEEE Journal on Selected areas in Communications, vol. 13, no. 7, pp. 1341-1347, 1995. Article (CrossRef Link)

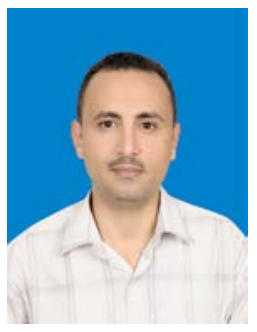

Y. A. Al-Gumaei received B.S. degree in electronic engineering in 2003 from IBB University, IBB, Yemen, and M.Eng.Sc degree in communication in 2010 from University of Malaya, Kuala Lumpur, Malaysia. He is currently working in his PhD degree in wireless cognitive radios and femtocell networks. His current research interest includes: resource allocation in cognitive radio, femtocell networks, game theory application in wireless networks.

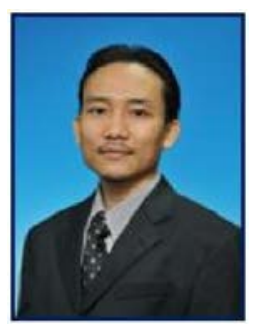

Kamarul Ariffin Noordin received his B.Eng.(Hons.) and M.Eng.Sc from University of Malaya, Kuala Lumpur, Malaysia in 1998 and 2001 respectively, and his PhD from Lancaster University in 2009, UK. He is currently an associate professor in Department of Electrical Engineering, University of Malaya, Kuala Lumpur, Malaysia. His current research interest includes resource allocation in wireless networks, Cognitive MAC for Heterogeneous Wireless Access Networks.

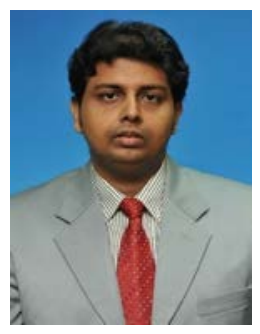

Ahmed Wasif Reza (Ph.D., M.Eng.Sc., B.Sc.Eng (Hons.), CEng (UK)) is currently working as a Senior Lecturer in University of Malaya, Faculty of Engineering, Department of Electrical Engineering, Malaysia. He has been working in the field of radio frequency identification (RFID), radio wave propagation, wireless sensor network, wireless communications, biomedical image processing, and cognitive radio and electromagnetic research, both in industrial exposure and academically research valued work. He has authored and co-authored a number of Science Citation Index (SCI) journals and conference papers (more than 100 papers). He has also participated as a reviewer and a committee member of a number of SCI/ISI journals and conferences.

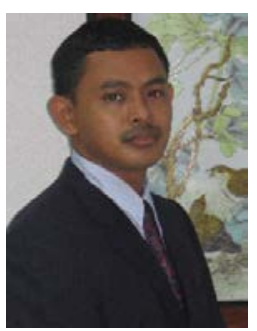

Kaharudin Dimyati received his bachelor degree from the University of Malaya, Malaysia in 1992. He then pursued for a PhD degree at the University of Wales Swansea, United Kingdom and awarded the degree in 1996. After completing his PhD in 1996, he joined University of Malaysia as a lecturer and later promoted to Associate Professor and Professor. He is now a professor at the National Defence University of Malaysia (NDUM), Malaysia. He joined NDUM in 2011. He is active in research, supervising research students and has published more than 150 papers. 